\title{
Lipid Membrane Based Biosensors
}

\author{
Georgia-Paraskevi Nikoleli ${ }^{1}$, Dimitrios P. Nikolelis ${ }^{2, *}$, \\ Christina G. Siontorou ${ }^{3}$, Marianna-Thalia Nikolelis ${ }^{2}$, \\ Stephanos Karapetis ${ }^{1}$
}

${ }^{1}$ Laboratory of Inorganic \& Analytical Chemistry, School of Chemical Engineering, Dept 1, Chemical Sciences, National Technical University of Athens, 9 Iroon Polytechniou St., Athens 157 80, Greece

${ }^{2}$ Laboratory of Environmental Chemistry, Department of Chemistry, University of Athens, Panepistimiopolis-Kouponia, GR-15771 Athens, Greece

${ }^{3}$ Laboratory of Simulation of Industrial Processes, Department of Industrial Management and Technology, School of Maritime and Industry, University of Piraeus

*To whom correspondence should be addressed

Email: nikolelis@chem.uoa.gr

\begin{abstract}
The exploitation of lipid membranes in biosensors has provided the ability to reconstitute a considerable part of their functionality to detect trace of food toxicants and environmental pollutants. Nanotechnology enabled sensor miniaturization and extended the range of biological moieties that could be immobilized within a lipid bilayer device. This chapter reviews recent progress in biosensor technologies based on lipid membranes suitable for environmental applications and food quality monitoring. Numerous biosensing
\end{abstract}


applications are presented, putting emphasis on novel systems, new sensing techniques and nanotechnology-based transduction schemes. The range of analytes that can be currently detected include, insecticides, pesticides, herbicides, metals, toxins, antibiotics, microorganisms, hormones, dioxins, etc. Technology limitations and future prospects are discussed, focused on the evaluation/ validation and eventually commercialization of the proposed sensors.

Keywords: biosensors, enzyme-based systems, receptor-based systems, toxins, food analysis, environmental monitoring, nanotechnology 


\section{Introduction}

A biosensor is a device that transforms the chemical information into an analytically useful signal. Biosensors usually contain two basic components connected in series: a biological recognition element ("receptor", i.e., enzyme, antibody, natural or artificial receptor, etc) and a physical transducer. The recognition system provides the chemical information (i.e., concentration of the analyte) into a physical output signal. The physical transducer alters the signal into electrical, optical, or piezoelectric, etc. output. The biological recognition element is in direct contact with a transduction element. Biosensors have made a large impact in the area of food and environmental applications and offer advantages in comparison to standard analytical techniques such as minimal sample preparation, real time detection, rapid response times, portability, high sensitivity and selectivity, etc. The term multiple-use biosensor is a device suitable for monitoring both the increase and decrease of the analyte concentration, whereas a single-use biosensor is a device which cannot be regenerated and is used only for one analysis test.

Recent progress in nanotechnology has given the opportunity to mass produce affordable devices and to integrate these into systems for market applications for environmental or food toxicants monitoring. These applications include a wide range of food toxicants and environmental pollutants, such as toxins, insecticides, pesticides, herbicides, microorganisms, bacteria, viruses and other microorganisms, polycyclic aromatic hydrocarbons (PAHs), hydrazines, phenolic compounds, allergens, genetically modified foods, hormones, dioxins, etc. 
Lipids are amphiphilic molecules, which possess both hydrophilic head groups and hydrophobic chains. Since the discovery of model bilayer lipid membranes (BLM) by Mueller et al. [1], there have been various attempts to use lipid films to construct biosensor devices for applications in food toxicants detection or environmental pollutants monitoring. However, free standing BLMs were very fragile and therefore not suitable for long-term repeated use. They had low mechanical and electrical stability which was the main obstacle to their practical applications. However, recent advances in the preparation of stabilized lipid bilayer have resulted to preparing lipid membrane based devices for detection of a large diversion of toxicants and pollutants in real samples. Lipid membranes based biosensors represent an appropriate biocompatible structure with fast response times (on the order of a few seconds), high sensitivity (i.e., nanomolar detection limits) and selectivity, small size, and offers many advantages compared with the bulky analytical instrumentation such as liquid chromatographic units. Most of these biosensors are cost efficient, easy-to-use, fast responding portability, and are good alternative to the expensive, bulky, time consuming standard analytical methods (i.e., chromatographic techniques). The new generation of lipid membrane nanosensors has the potential for the development of site-specific monitors with respect to analytical performance, operational stability and response.

This work reviews the status of the various nanostructure lipid membrane based biosensors that are used to monitor food toxicants and environmental pollutants. The chapter provides the state of art of design and microfabrication of prototype lipid membrane nanosensing devices for the 
rapid in the field detection of food toxicants and environmental pollutants and the challenges that lie ahead.

\section{Methods for preparation biosensors based on lipid films}

A number of techniques have been described in the literature the last two decades for techniques for the preparation of stabilized lipid membranes that are not prone to electrical or mechanical breakage and provide devices that can be used for practical applications. Most of these techniques provide lipid membranes with a size is less than $1 \mu \mathrm{m}$ and therefore can be considered as nanosensors. These biosensors have been used for electrochemical experimentation and belong therefore in electrochemical biosensors. An exception is the development of stabilized polymerized lipid films on a filter paper that switch on and off their fluorescence and therefore belong to optical biosensors. Below we provide the various techniques for the preparation of biosensors based on lipid membranes and mainly those that are mini- or nanosensors.

\subsection{Metal supported lipid membranes}

A simple system for the preparation of stabilized bilayer lipid membrane (sBLM) at the freshly cut tip of Teflon coated metallic wire was reported by Tien and Salamon [2]. The technique of formation of sBLMs is 
based on the interaction of an amphiphatic lipid molecule with a nascent metallic surface. One end of a Teflon-coated stainless steel metal wire (with diameter of $0.1-0.5 \mathrm{~mm}$ ) was immersed in a lipid solution in chloroform and then, while still immersed in chloroform, the tip is cut off with a miniature guillotine. The fresh tip of the wire becomes coated with the lipid membrane and was placed in the electrolyte (usually $0.1 \mathrm{M} \mathrm{KCl}$ ), whereupon the lipid film spontaneously thinned, forming a self-assembled lipid bilayer membrane (sBLM). A Schematic of the sensor, measurement setup, and lipid selfassembly process is shown in Figure 1.

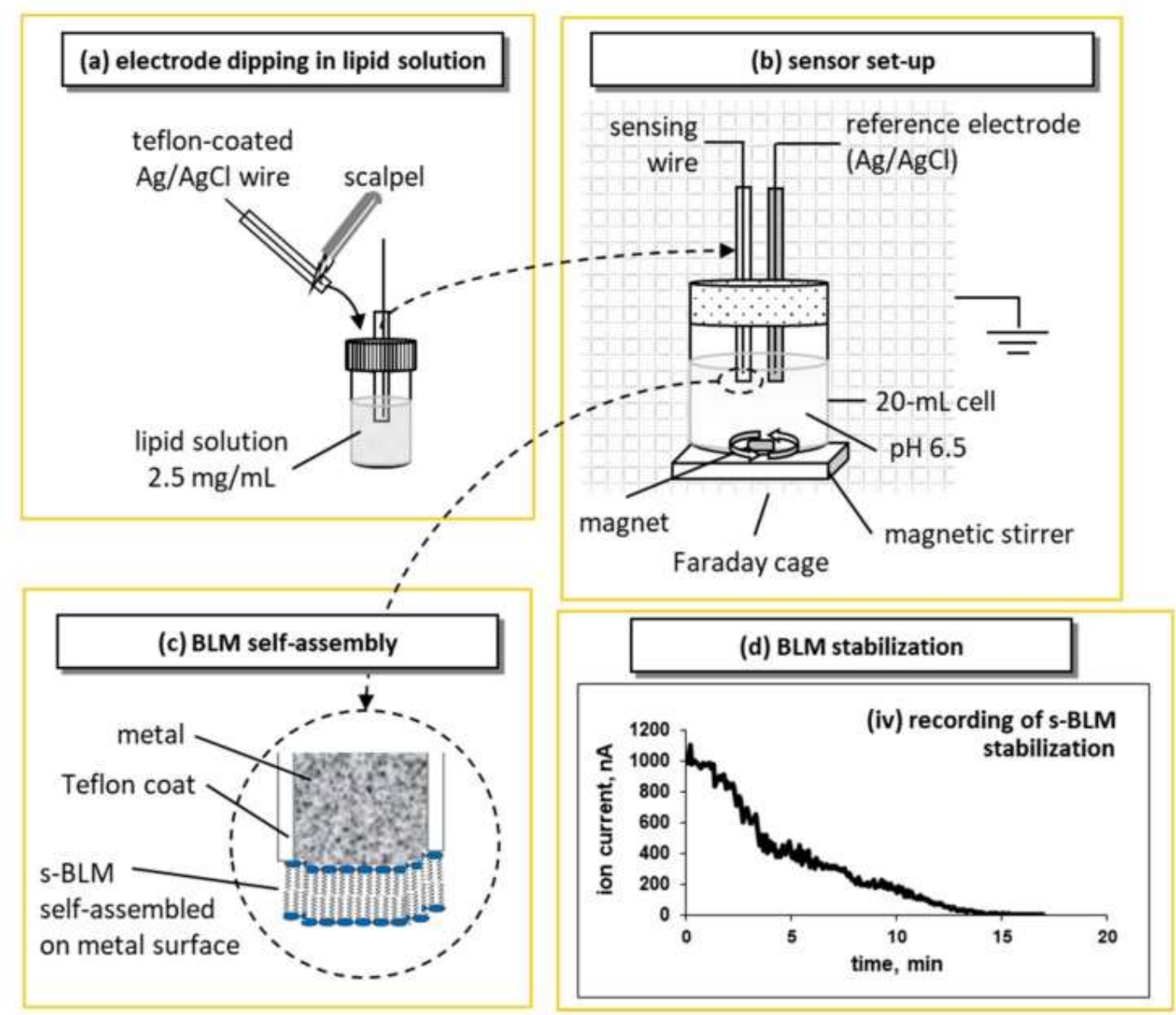

Figure 1. Schematic of the sensor, measurement setup, and lipid self-assembly process (not drawn to scale): (a) the sensing electrode is tipped with a scalpel and immediately immersed in lipid solution before dipped in the electrolyte solution. (b) 
The electrochemical setup consists of a $20-\mathrm{mL}$ cell and a two-electrode configuration, i.e., the sensing electrode and a $\mathrm{Ag} / \mathrm{AgCl}$ reference electrode, placed in a grounded Faraday cage; an external $\mathrm{DC}$ potential of $25 \mathrm{mV}$ is applied between the electrodes and the ionic current through the BLM is measured with a digital electrometer; the cell is stirred using a magnetic stirrer. (c) Upon immersion, the lipid droplet attached to the wire is self-assembled into a bilayer that has one layer adsorbed on the metal surface and the other facing the aqueous solution. (d) Recording of the ion current decrease during the self-assembly process; recording started at the immersion of the sensing electrode in the electrolyte solution [reprinted from ref. 3]..

sBLMs have been fully characterized [2,4,5]. The diameter and composition of the wires that were used were found to play an important role in the time for device stabilization, and on the magnitude and noise of the background ion current $[4,5]$. The use of a wire of $0.25 \mathrm{~mm}$ diameter and of decane as a solvent should be avoided because the lipid membrane is so called "black lipid membrane" and these films do not provide reproducible results. Silver wires of 0.5 and $1.0 \mathrm{~mm}$ diameter provide BLMs which were mechanically and electrical stable for over 48 hours.

Previous studies have provided a model of a potential profile across sBLMs and have evaluated the structure of the inner lipid layer (facing the silver wire support). It has been suggested that the lipid headgroups bind to the electrode by interactions of oxygen atoms of the phosphate groups of the lipids with silver ions in the metal lattice $[6,7]$. Note that the silver metal is not fully insulated from the chloride ion by a BLM. Furthermore, chloride ions can also readily move through the lipid film during the initial BLM stabilization 
process and chloride would react with the silver metal to form silver chloride [4,5]. Potentiometric (against a $\mathrm{Ag} / \mathrm{AgCl}$ reference electrode) experiments [5] show only small voltages (relative to a silver wire against a $\mathrm{Ag} / \mathrm{AgCl}$ reference electrode) when using the surface of a silver $\int$ wire after BLM stabilization and BLM removal with an organic solvent rinse. These results suggest that the surface of the metal is likely coated with a thin layer of silver chloride and that the sBLM consists from smaller BLMs on the order of $\mathrm{nm}$ which provides a nanostructure in these devices [8].

\subsection{Stabilized lipid films formed on a glass fiber filter}

The preparation of stabilized lipid membranes supported on ultrafiltration glass fiber filters has been reported in the literature [9]. These supported lipid films on ultrafiltration glass fiber filters have allowed the practical application in real samples, e.g. for the determination of aflatoxin $M_{1}$ in milk and milk preparations [10]. The lipid membrane is formed on a microporous filter glass fiber disk $[9,10]$. The filters and (nominal) pore size used were GF/F glass microfiber, $0.7 \mu \mathrm{m}$ (Whatman ScientificLtd., Kent, U.K.).

The experimental set up which is used for the formation of these stabilized BLMs consists of two plexiglas chambers separated by a SaranWrap partition (thickness of ca. $10 \mu \mathrm{m}$ ). This plastic partition was cut to more than twice the size of the contact area of the faces of the chambers and was folded in half; then, a hole (with diameter $0.32 \mathrm{~mm}$ ) was punched through the double layer of the plastic film with a perforation tool. A microporous glass 
GF/F microfiber disk (diameter of ca. $0.9 \mathrm{~cm}$ and nominal pore size of $0.7 \mu \mathrm{m}$ ) is placed between the two plastic layers, centered on the $0.32 \mathrm{~mm}$ orifice. The partition containing the filter membrane was then clamped between the two plexiglas chambers. One of the chambers consisting an electrochemical cell, had a circular shape (diameter $1.0 \mathrm{~cm}$ and depth $0.5 \mathrm{~cm}$ ); this chamber was connected with plastic tubing which was used for the flow of the carrier solution. An $\mathrm{Ag} / \mathrm{AgCl}$ reference electrode was immersed in the waste of the carrier electrolyte solution. The second chamber was cylindrical and had its longitudinal axis perpendicular to the flow of the carrier solution. The upper hole of this cell was circular (surface area of about $0.2 \mathrm{~cm}^{2}$ ) and the lower was elliptical (with diameters 0.5 and $1.4 \mathrm{~cm}$ parallel and vertical to the flow of the carrier electrolyte solution, respectively). The lower hole was facing the opposing cell. An $\mathrm{Ag} / \mathrm{AgCl}$ reference electrode was positioned the center of the cylindrical cell. An external voltage of 25 or $50 \mathrm{mV}$ d.c. is applied between the two reference electrodes. A Keithley digital electrometer is used as a current-to-voltage converter. A peristaltic pump is used for the flow of the carrier electrolyte. Injections of the samples are made with a Hamilton repeating dispenser. The electrochemical cell and electronic equipment were isolated in a grounded Faraday cage. A simple scheme of the apparatus used is presented in Fig. 2. The procedure for the formation of the stabilized BLMs is as follows: [9,10]: Lipid solution (ca. $10 \mu \mathrm{L}$ ) is added dropwise from a microliter syringe to the water surface in the cylindrical cell near the plastic partition. The level of the electrolyte solution is dropped below the the 0.32 $\mathrm{mm}$ orifice and then raised again; this procedure should take place within a 
few seconds. The formation of the BLMs has to be verified by the ion current magnitude and by the electrochemical characterization using gramicidin D.

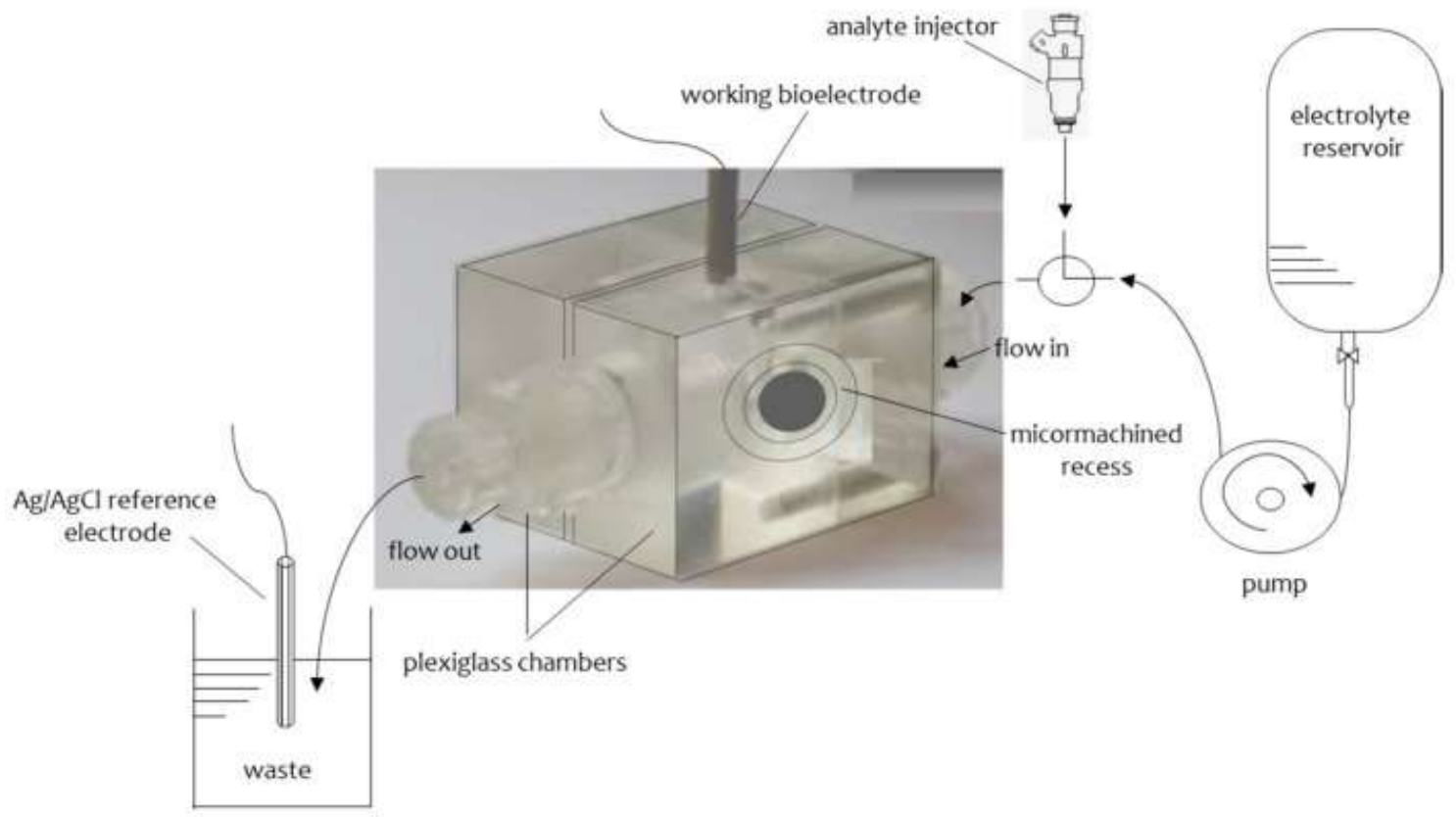

Figure 2. Schematic of the experimental set-up; the micromachined chambers are separated by a thin (12.5 $\mu \mathrm{m}$ thick) polyvinylidene chloride wrap and enclose the microfiber disk. For more details, see text.[from ref. 3]

\subsection{Polymer-supported bilayer lipid membranes}

The preparation of polymer stabilized was recently described in the literature' The construction of these lipid membrane based biosensors took place by using UV irradiation instead of heating the lipid mixture to $60^{\circ} \mathrm{C}$ $[11,12]$ Thus this process retaines the activity of an enzyme (i.e., acetylcholinesterase), whereas heating can deactivate it. The results indicated that the polymerization is completed within 4 hours by using physicochemical 
methods such as DSC, IR or Raman spectrophotometry. The preparation of these devices makes possible the practical use of biosensors based on lipid membranes for chemical sensing, because it allows incorporation of a "receptor" molecule such as enzyme, antibody or ion-channel receptor in these lipid films and more importantly these devices are stable outside of the solution in the air for more than $48 \mathrm{~h}$.

The preparation of these stabilized lipid films is as follows [11,12]: Briefly, $0.8 \mathrm{~mL}$ of a mixture containing $4 \% \mathrm{w} / \mathrm{v}$ egg phosphatidylcholine (PC) in $\mathrm{n}$ hexane (this solvent evaporates so that these films do not retain the solvent in their structure) were mixed with $0.07 \mathrm{~mL}$ of methacrylic acid, $0.8 \mathrm{~mL}$ of ethylene glycol dimethacrylate, $8 \mathrm{mg}$ of 2,2'-azobis-(2-methylpropionitrile) and $1.0 \mathrm{~mL}$ of acetonitrile. The mixture was sparged with nitrogen for about $1 \mathrm{~min}$ and sonicated for $30 \mathrm{~min}$. For the preparation of the stabilized lipid films, $0.15 \mathrm{~mL}$ of this mixture was spread on a microfilter (microporous glass GF/F microfiber disk with a diameter of ca. $0.9 \mathrm{~cm}$ and nominal pore size of $0.7 \mu \mathrm{m})$. The filter with the mixture was then irradiated using the UV deuterium lamp. Raman spectrometry and differential scanning calorimetry (DSC) were used to monitor the kinetics of the polymerization process. The measuring set up was similar to that presented on Fig. 2. These membranes were stable in storage in air for repetitive uses. 


\subsection{Polymer lipid films supported on graphene microelectrodes}

Graphene nanomaterials have received tremendous interest in the field of basic research and in technological applications due to their unique physicochemical properties, i.e., good sensing ability, and excellent mechanical, thermal and electrical properties; other advantages include large surface-to-volume ratio, excellent biocompatibility, high electron-transfer rates, non-toxicity and bio-safety. The development of biosensors is an interesting application of this field of nanotechnology. Their implementation in electrochemical biosensing is quite beneficial as the large surface-area-tovolume ratio enables miniaturization, increases speed of response and allows for lower detectabilities while solving the biocompatibility and biofouling problems. Several examples in the development of nanobiosensors by integrating enzymes and antibodies were recently described in literature. Stabilized lipid films were wrapped around a copper wire containing graphene nanosheets $[13,14]$. These nanosensors have been implemented in the rapid detection of food toxicants, environmental pollutants and toxins in real samples, such insecticides [14], naphthalene acetic acid [15], cholera toxin [16], and saxitoxin [17].

The preparation of graphene microelectrodes was as follows [13-17]: A homogeneous graphene dispersion $(\sim 0.4 \mathrm{mg} / \mathrm{mL})$ has been obtained in $N$ methyl-pyrrolidone (NMP) through mild sonication for 180 hours and centrifugation at $700 \mathrm{rpm}$ for $2 \mathrm{~h}$. This suspension has been poured onto a copper wire $(d=0.25 \mathrm{~mm})$ mounted on a glass fiber filter and evaporation of 
the organic solvent has been carried out using a fan heater. This copper wire has been utilized to establish the connection for the extraction of voltage signals for the calibration curve. Thus, a simplistic approach of drop wise dispersion of graphene suspended in NMP solution has been utilized to scatter the graphene nanosheets on the copper wire. The extended sonication time results in a good fraction of monolayer sheets but with smaller lateral sizes.

The procedure of construction of these devices is in brief as follows [13-17]: Stabilized lipid films were prepared by polymerization with a procedure similar to that previously described [11,12]. Briefly, $0.15 \mathrm{~mL}$ of a mixture containing $5 \mathrm{mg}$ of a mixed lipid powder composed of $35(\mathrm{w} / \mathrm{w})$ DPPA and $65(\mathrm{w} / \mathrm{w})$ of DPPC (1.75 mg DPPA and $3.25 \mathrm{mg}$ DPPC) were mixed with $0.070 \mathrm{~mL}$ of methacrylic acid, $0.8 \mathrm{~mL}$ of ethylene glycol dimethacrylate, $8 \mathrm{mg}$ of 2,2'-azobis-(2-methylpropionitrile) and $1.0 \mathrm{~mL}$ of acetonitrile. DPPC is used as lipid and not PC which can be oxidized by air and does not provide reproducible results The mixture was spumed with nitrogen for about $1 \mathrm{~min}$ and sonicated for $30 \mathrm{~min}$. This mixture could be stored in the refrigerator. For the preparation of the stabilized lipid films, $0.15 \mathrm{~mL}$ of this mixture was spread on the glass filer microfilter. The filter with the mixture was then irradiated using a UV deuterium lamp. Raman spectrometry was used to monitor the kinetics of the polymerization process $[11,12]$.

The enzyme, antibody or receptor ("receptor") were incorporated in these BLMs prior polymerization by spreading $15 \mu \mathrm{L}$ of the "receptor" suspension with the polymerization mixture (i.e., for the preparation of the 
stabilized lipid films, $0.15 \mathrm{~mL}$ of the polymerization mixture and $15 \mu \mathrm{L}$ of "receptor" suspension were spread on the microfilter). The preparation of the potentiometric biosensor has been finalized by encapsulation of the filtersupported polymerized lipid film onto the copper wire containing graphene nanosheets.

\section{Applications of lipid film based biosensors in food analysis and environmental monitoring}

The stabilized supported lipid membranes biosensors were used for the flow injection analysis (FIA) of pesticides [18]. Carbofuran was chosen as a typical pesticide. The determination of the pesticide is based on the degree of inhibition and reactivation of enzyme by injections of substrate. Carbofuran was determined at concentration levels of $10^{-7}$ to $10^{-9} \mathrm{M}$. The investigation of the effect of interferences included compounds usually found in foods, proteins and lipids. The results have shown no interferences from these compounds. The technique was applied in various real samples of fruits, vegetables and dairy products. The recovery ranged between ca. 96 and $106 \%$ which shows no interferences from the matrix effects.

A paper was reported in the literature using a synthetic "receptor" immobilized on supported lipid films on glass fiber filters. The supported lipid films were modified by calixarenes and permitted sensitive and rapid determination of insecticides in fruits and vegetables [19]. Other applications in this field include the construction of a disposable chemosensor for the selective 
fast detection of food hormones (i.e., naphthalene acetic acid) in fruits and vegetables [20] and of zinc in waters [21].

A potentiometric urea lipid film based minisensor on graphene nanosheets in which a polymeric lipid membrane was deposited has been recently reported in the literature [22]. The structural characteristics of graphene nanosheets have been studied through atomic force microscopy (AFM) and transmission electron microscopy (TEM) measurements. UV-Vis and Fourrier transform IR (FTIR) spectroscopy have been utilized to study the pre- and postconjugated surfaces of graphene nanosheets. The presented potentiometric urea biosensor (Figure 3) exhibits good reproducibility, reusability, selectivity, fast response times $(\sim 4 s)$, long shelf life and high sensitivity having a slope of ca. $70 \mathrm{mV} /$ decade over the urea logarithmic concentration range from $1 \times 10^{-6} \mathrm{M}$ to $1 \times 10^{-3} \mathrm{M}$.

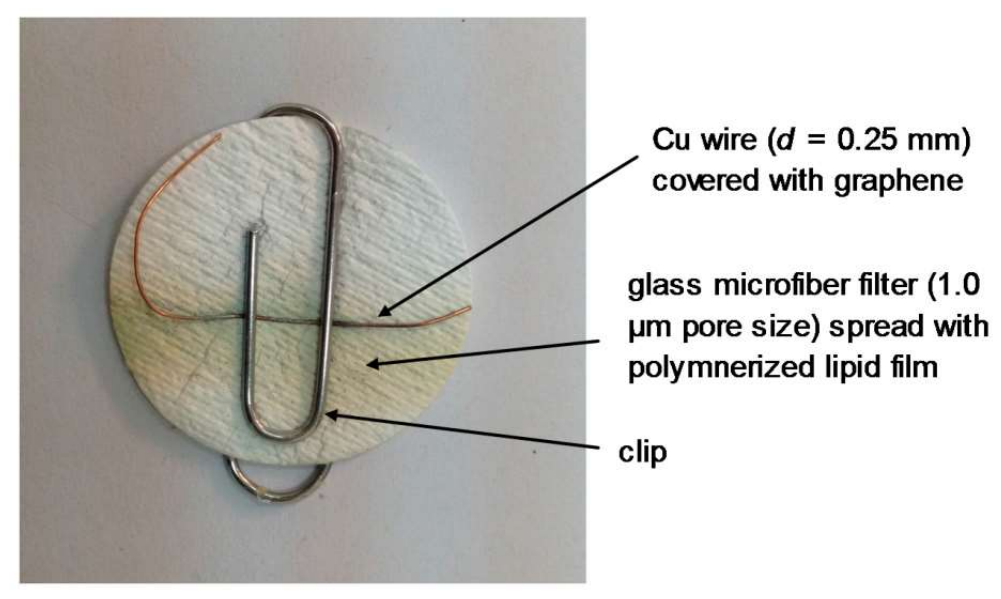

Figure 3. Schematic of a lipid membrane based biosensor on graphene electrode. This device was used for the potentiometric determination of urea (reprinted from reference 23). 
The electrochemical interactions of naphthalene acetic acid (NAA) with stabilized lipid films supported on a methacrylate polymer on a glass fiber filter with incorporated auxin-binding protein 1 receptor were investigated with a purpose to develop a nanosensor for the rapid determination of NAA in fruits and vegetables [24]. A FIA technique was used; NAA was injected into the flowing streams of a carrier electrolyte solution, the flow of the electrolyte solution stops and an ion current transient was obtained; the peak height of which was correlated to the hormone concentration (with $\mu \mathrm{M}$ detection limits). The response times were rapid (on the order of $5 \mathrm{~min}$ ). The effect of interferences included a wide range of compounds. The results showed no interferences from these compounds in concentration levels usually found in real samples. The method was applied for the determination of NAA in fruits and vegetables and the reproducibility of the method was satisfactory. Thus, a quantitative method for the detection of NAA in fruits and vegetables that can be complimentary to HPLC methods was attained.

A potentiometric carbofuran minisensor on graphene nanosheets with incorporated lipid membranes has been described in the literature [25]. The graphene electrode was used to develop a selective and sensitive chemical sensor for the detection of carbofuran by incorporating an artificial selective receptor (resorcin[4]arene receptor) on stable lipid films. This chemical minisensor can determine carbofuran concentrations at $\mathrm{nM}$ concentration range, with rapid response times of ca. $20 \mathrm{~s}$, easy to construct and exhibits good reproducibility, reusability, selectivity, long shelf life and high electrode slope of ca. $59 \mathrm{mV} /$ decade over the carbofuran logarithmic concentration range from $10^{-6}$ to $10^{-3} \mathrm{M}$. 
A work that explores the interactions of atrazine with bilayer lipid films that can be used for the direct electrochemical determination of this herbicide has been described in the literature [26]. The interactions of atrazine with solventless bilayer lipid membranes (BLMs) were found to be electrochemically transduced by these films in the form of a transient current signal with duration of $\mathrm{s}$ and reproducibly appeared within $1 \mathrm{~min}$ after exposure of the membranes to atrazine. The sensitivity of the response was maximized by the use of BLMs composed of $35 \%$ (w./w.) DPPA, and by alteration of the phase distribution within membranes by the introduction of calcium ions in bulk solution. The hydrogen bonding between atrazine and the carbonyl group of the lipid was investigated by the addition of plateletactivating factor (PAF; an ether analog of PC) in BLMs composed of phosphatidylcholine. The peak height (i.e., current) was linearly related to the concentration of this pesticide in bulk solution with $\mu M$ detection limits.

A work that describes a method for the rapid and sensitive electrochemical flow injection monitoring (FIA) and analysis of mixtures of the triazine herbicides simazine, atrazine and propazine using stabilized systems of filter-supported BLMs consisted of egg PC and DPPA has been described in the literature [27]. Injections of these herbicides were made into flowing streams of a carrier electrolyte solution and a transient current signal with a duration of seconds reproducibly appeared in less than two min after exposure of the lipid membranes to the herbicides. The magnitude of the peak height was linearly related to the concentration of the herbicide, which could be determined at $\mu M$ range. Repetitive cycles of injection of herbicides have shown no signal degradation during each cycle. The time of appearance of 
the transient signal was different for each triazine and increased to the order of simazine, atrazine and propazine which has allowed selective detection and analysis of these triazines in mixtures.

A strategy was described in the literature that was based on monitoring of changes of ion current through a lipid film with immobilized DNA probes caused by interaction of these lipid membranes with hydrazine compounds [28]. A s-BLM that was consisted of egg PC was deposited on a silver metal electrode. The oligomers used were single stranded deoxyribonucleic acids: thymidylic acid icosanucleotide terminated with a C-16 alkyl chain to assist incorporation into s-BLMs $\left(\mathrm{dT}_{20}-\mathrm{C}_{16}\right)$, and deoxyadenylic acid icosanucleotide $\left(d_{20}\right)$. These s-BLMs with incorporated DNA interact with hydrazines, and it is possible to monitor ppb levels of hydrazine compounds (i.e., hydrazine, methylhydrazine, dimethylhydrazine and phenylhydrazine). This BLM/DNA biosensor offers a highly sensitive, selective, fast, and portable biosensor for monitoring these environmentally and toxicologically significant compounds.

A paper appeared in the literature that describes the investigations of electrochemical interactions of cholera toxin with stabilized lipid films on a polymer over a glass fiber Whatman GF/F filter with incorporated ganglioside GM1; this has lead to the development of a minisensor for cholera toxin [29]. The analyte was injected into the flowing streams of a carrier electrolyte solution, the flow of the solution stopped for $5 \mathrm{~min}$ and an ion current transient was obtained, the peak height of this transient was correlated to the cholera toxin concentration and had detection limits of $0.06 \mu \mathrm{M}$. Further work is 
directed to investigate the rapid detection of other toxins used in bioterrorism and uses this novel ultrathin film technology.

A potentiometric cholera toxin minisensor on graphene nanosheets with incorporated lipid films has been described in the literature [30]. Ganglioside GM1 (a natural cholera toxin receptor) was incorporated on stabilized lipid films on graphene electrodes, providing adequate selectivity for the detection of cholera toxin over a wide range of concentrations, rapid response time of ca. $5 \mathrm{~min}$, and detection limits of $1 \mathrm{nM}$. The proposed sensor is easy to construct and exhibits good reproducibility, reusability, selectivity, long shelf life and having a slope of ca. $60 \mathrm{mV} /$ decade of toxin concentration. The method was evaluated, implemented and validated in lake water samples. This novel ultrathin film technology is currently adapted to the rapid detection of other toxins and could be used as a weapon against bioterrorism.

A novel electrochemical biosensor based on a supported polymeric lipid membranes with immobilized Sheep anti-PCB antibody for the rapid determination of arochlor 1242 in flowing solution streams (FIA systems) has been described in the literature [31]. This antibody was immobilized in the lipid membrane during polymerization of the film; the injections of antigen were made into flowing streams of a carrier electrolyte solution. The experimentation was made in a stopped-flow mode; the lipid mixtures were composed of $15 \%(w / w)$ PA and $85 \%$ of DPPC to provide only one and only single transient current signal with a peak height that was related to the antigen concentration. Lipid films that were composed of $35 \%$ DPPA were used to investigate the regeneration of the active sites of antibody after complex formation; this was succeeded by continuous washing with the 
carrier electrolyte solution. Repetitive cycles of injection of antigen have exhibited that the maximum number of cycles is ca. 5 .

A potentiometric saxitoxin minisensor based on graphene nanosheets with incorporated lipid films and immobilized anti-STX (which is the natural saxitoxin receptor) on stabilized lipid films was recently reported in the literature [32]. A good selectivity and sensitivity for the detection of saxitoxin, fast response times of ca. 5-20 min, and detection limits of $1 \mathrm{nM}$ were observed. The proposed minisensor is easy to construct and exhibits good reproducibility, reusability, selectivity, long shelf life and having a slope of ca. $60 \mathrm{mV} /$ decade over saxitoxin concentration. The method was evaluated and validated in lake water and shellfish samples. This novel ultrathin film technology is currently adapted to the rapid detection of other toxins that could be used as weapons against bioterrorism.

An electrochemical biosensor that is suitable for the rapid and sensitive screening of the sweetener sucralose based on surface-stabilized bilayer lipid membranes (s-BLMs) composed of PC was recently described in the literature [33]. The interactions of sucralose with s-BLMs provided an ion current increase, that appeared within a few seconds after exposure of the membranes to the sweetener. The mechanism of signal generation was explored by DSC studies. The mechanism was found to be related to changes of the electrostatic fields of the lipid membrane. These studies have shown that there is an increase of the molecular area of the lipids at the membranes and a stabilization of a gel phase structure; this was due to adsorption of the sweetener in the membrane surface. The current signal increases were correlated to the concentration of sucralose in bulk solution in the $\mu \mathrm{M}$ 
concentration range. The present lipid film based biosensor has provided a rapid response (order of seconds) to alterations of sucralose concentration (5$50 \mu \mathrm{M})$ in the bulk solution. The electrochemical transduction of interactions of this sweetener with s-BLMs was evaluated by its determination in granulated sugar substitute products.

A method that reports the FIA and analysis of mixtures of the artificial sweeteners acesulfame-K, cyclamate, and saccharin using stabilized systems of filter-supported BLMs was described in the literature [34]. Injections of artificial sweeteners were made into flowing streams of a carrier electrolyte solution, and a transient current with duration of seconds appeared in less than $1 \mathrm{~min}$ after exposure of the lipid membranes to the artificial sweeteners. The peak height of this signal was linearly related to the concentration of artificial sweeteners, which could be determined at $\mu \mathrm{M}$ concentration range. The repetitive cycles of injectionsprior to signal degradation was 30 . The time of appearance of the transient response was different for each artificial sweetener and increased in the order of cyclamic acid, acesulfame- $\mathrm{K}$, and saccharin. The difference in time of response has allowed selective detection and analysis of these artificial sweeteners in mixtures. The effect of interferences usually found in foods, proteins, and lipids was investigated. The results showed no interferences from these constituents of real food samples. The method was applied in real food samples (i.e., artificial sweetener tablets, diet soft drinks, wines, and yogurts) that contain mixtures of these artificial sweeteners with aspartame and other compounds. A comparison of results using the present method and that of an Official Method of Analysis showed good agreement between the two methods. 
Investigation of the transport phenomena through channels/pores it is very important for various biological, medical, and technical applications. The scope of a paper appeared in the literature is the development of nanofluidics for the creation of biosensors capable of detecting single molecules and manipulating them [35]. The detection of molecules was based on the measurement of the current through a channel when a molecule enters the channel, which has a diameter comparable with the molecule size, the current reduces. In order to improve transport properties of such channels, their walls are often coated with a lipid bilayer, which behaves as twodimensional liquid and thus is capable of supporting transport phenomena. Presently, this property of lipid membranes was utilized for the development of a technique for detecting and controlling transport of single-stranded DNA through channels formed by membrane cylinders with the luminal radii of 5-7 $\mathrm{nm}$. It was demonstrated that in the conditions of small ion strength, the appearance of a DNA molecule inside such channel is accompanied by an increase of its ion conductivity and can be controlled by the polarity of the applied voltage. The peak height of the current increase permits to evaluate the number of DNA molecules inside the channels. It was also demonstrated that upon adsorption of DNA molecules on the lipid bilayer surface, the membrane cylinder behaves as a voltage-sensitive selective ion channel.

Eggshell was used as a biomembrane for immobilization of urease for the development of a potentiometric urea biosensor [36]. Eggshell membrane was treated with polyethyleneimine (PEI) to impart polycation characteristics. Urease was immobilized on the PEI treated eggshell membrane through adsorption. SEM study was carried out to observe the changes 
in surface morphology after immobilization. FTIR study of membrane was carried out to observe the changes in IR spectra after immobilization of the enzyme. The biosensor has shown a sigmoidal response for the urea concentration range from 0.5 to $10 \mathrm{mM}$. The response time of the biosensor was $120 \mathrm{~s}$. One membrane could be was reused for 270 reactions without loss of activity. The urease-eggshell membranes were stable for 2 months when stored in buffer even at room temperature.

Bilayer lipid membranes (BLMs) can be successfully formed on polymers electrodeposited on a solid metallic support. Avidin-biotin interactions were employed for immobilisation of the enzyme on the surface of the BLM. Improved long-term stability and better selectivity towards certain interfering electroactive species were observed for a BLM glucose biosensor based on glucose oxidase immobilised on a platinum support modified with any of several polymers [37]. However, the best results were obtained for the mediated system in which the BLM was formed on a Pt support covered with a layer of evaporated Nafion with incorporated ferrocene. The stable and sensitive response with significant elimination of the influence of electroactive interferences on the signal magnitude should allow a practical application of such miniaturised biosensors.

A novel chemiluminescence biosensor based on a supported lipid layer incorporated with ganglioside GM1 was reported for the detection of cholera toxin (CT). The planar supported lipid membrane was prepared as biosensing interface via spontaneous spread of ganglioside-incorporated phospholipid vesicles on the octadecanethiol-coated gold surface [38]. The specific interaction of multivalent $\mathrm{CT}$ by ganglioside GM1 molecules enables the 
biosensor to be implemented via a sandwiched format using a liposome probe functionalized with GM1 and horseradish peroxidase (HRP). Then, the presence of the target CT could be determined via the HRP-catalyzed enhanced chemiluminescence reaction. The developed strategy offers several unique advantages over conventional biosensors in that it allows for an easy construction and renewal of the sensing interface, a small background signal due to low non-specific adsorption of serum constituents on the lipid membrane, and effective immobilization of multiple biocatalytic amplifiers and recognition components via common phospholipid reagents. The developed biosensor was shown to give chemiluminescence signal in linear correlation to CT concentration within the range from $1 \mathrm{pg} \mathrm{mL}^{-1}$ to $1 \mathrm{ng} \mathrm{mL}^{-1}$ with readily achievable detection limit of $0.8 \mathrm{pg} \mathrm{mL}^{-1}$.

A work that reports a novel bilayer lipid membranes (BLMs) nucleic acid biosensor supported by modified patch-clamp pipette electrode was developed to detect staphylococcus enterotoxins B (SEB) gene [39]. Hydrophobic dodecane tail $\left(\mathrm{C}_{12}\right)$ modified $18 \mathrm{bp}$ single-stranded DNA (ssDNA) probe was immobilized on BLMs. The electrochemical currents versus the different concentration of SsDNA probe immobilized on BLMs indicated linear correlation. The BLMs nucleic acid biosensor was fabricated by selecting the ssDNA probe as the signal sensing element with the concentration of $273.65 \mathrm{ng} / \mathrm{mL}$. The electrochemical performance of the biosensor for the detection of SEB was explored. The result showed that a linear relationship existed between the current and In(concentration) from 20 to $5000 \mathrm{ng} / \mathrm{mL}$ and the detection limit was $20 \mathrm{ng} / \mathrm{mL}$. In addition, the biosensor has shown a specific response to SEB gene and no significant 
current alteration in electrolyte which containing no SEB gene. Atom Force Microscope (AFM) images could be observed and used to evaluate the superficial microstructure of BLMs, ssDNA immobilized on BLMs and BLMs after hybridization. The BLMs nucleic acid biosensor supported by modified patch-clamp pipette electrode will become a highly sensitive, rapid, selective analytical tool for detection of Staphylococcus aureus, which produce SEB.

A nanostructure electrochemical biosensor was developed to directly detect and screen estrogenic substances based on estrogen receptor (ER) binding without the use of radio- or enzyme-labeled compounds [40]. The biosensor was fabricated by immobilization of ERs in s-BLM modified with Au nanoparticles, and the properties of the modified electrodes were characterized by cyclic voltammetry and impedance spectroscopy. The results have shown that the biosensor was able to detect the natural estrogen $17 \beta-$ estradiol with an acceptable linear correlation ranging from 5 to $150 \mathrm{ng} / \mathrm{L}$ and a detection limit of $1 \mathrm{ng} / \mathrm{L}$. The biosensor could also detect other known xenoestrogens such as bisphenol A and 4-nonylphenol with satisfied sensitivity and quantitative results. The biosensor had good reliability and repeatability, and the Au nanoparticles greatly enhanced the sensitivity and stability of the biosensor. Moreover, estrogenic activity of water samples determined by this biosensor was in good agreement with that determined by MCF-7 cell proliferation assay. 


\section{Conclusions and future prospects}

The present paper provides various ways for the preparation of nanosensors based on a lipid film technology for food and environmental analytical applications. Recent technological advances are the construction of stabilized supported lipid film on graphene nanoelectrodes with an incorporated "receptor" (enzyme, antibod or natural or artificial receptor) stable in air that can be portable for in the field applications. These sensors reveal detection limits in the $\mathrm{nM}$ concentration range. The most important aspect of the present efforts is to provide a portable unit that can be used for in-field and market applications and that also can be commercialized

The results have shown that a diversity of lipid film based nanosensors can be reused after storage in air even after a period of a couple of months, and can be reproducibly fabricated with simplicity and low cost. These nanosensors have fast response times, are easy to construct and have a lower cost than that based on chromatographic techniques; they also can be used as rapid portable detectors complimentary to these methods for in-field and market measurements in foods and for environmental monitoring.

The present review describes biosensors based on lipid film technology that can be used for the rapid detection of food toxicants and environmental pollutants such as toxins, carbamates, hormones, polycyclic aromatic hydrocarbons, etc and highlights their advantages which are high sensitivity and selectivity, rapid response times, portability, etc. It is of common sense that the use of nanotechnology to construct lipid membrane based biosensors will provide devices with even improved characteristics. 


\section{Author Contributions}

All authors contributed equally to this work.

Conflicts of Interest

The authors declare no conflict of interest.

\section{References}

1. Mueller, P., Rudin, D.O., Tien, H.T., Wescott, W.C. Reconstitution of cell membrane structure in vitro and its transformation into an excitable system, Nature 1962, 194, 979-980.

2. Tien, H.T.; Salamon, Z. Formation of self-assembled lipid bilayers on solid substrates. J. Electroanal. Chem. Interfacial Electrochem. 1989, 22, 211218.

3. Nikoleli, G.-P., Nikolelis, D., Siontorou, C.G., Karapetis, S. Lipid membrane nanosensors for environmental monitoring: The art, the opportunities, and the challenges. Sensors 2018, 18(1), 284;

4. Nikolelis, D.P.; Siontorou, C.G.; Krull, U.J.; Katrivanos, P.L. Ammonium ion minisensors from self-assembled bilayer lipid membranes using gramicidin as an ionophore. Modulation of ammonium selectivity by platelet-activating factor. Anal. Chem. 1996, 15, 1735-1741.

5. Siontorou, C.G.; Nikolelis, D.P.; Krull, U.J.; Chiang, K.L. A triazine herbicide minisensor based on surface-stabilized bilayer lipid membranes. Anal. Chem.1997, 69, 3109-3114. 
6. Hianik, T.; Dlugopolsky, J.; Gyepessova, M. Electrostriction of lipid bilayers on a solid support. Influence of hydrocarbon solvent and d.c. voltage. Bioelectrochem. Bioenerg. 1993, 31, 99-111.

7. Hianik, T.; Passechnik, V.I.; Sargent, D.F.; Dlugopolsky, J.; Sokolikova, L. Surface potentials and solvent redistribution may explain the dependence of electrical and mechanical properties of supported lipid bilayers on applied potential and bilayer history. Bioelectrochem. Bioenerg. 1995, 37, 61-68.

8. Passechnik, V.I.; Hianik, T.; Ivanov, S.A.; Sivak, B. Specific capacitance of metal supported lipid membranes. Electroanalysis 1998, 10, 295-302.

9. Nikolelis D.P., Siontorou C.G., Andreou V.G., Krull U.J. Stabilized bilayerlipid membranes for flow-through experiments. Electroanalysis. 1995, 7, $531-536$.

10. Andreou V.G., Nikolelis D.P. Flow injection monitoring of aflatoxin $M_{1}$ in milk and milk preparations using filter-supported bilayer lipid membranes. Anal. Chem. 1998, 70, 2366-2371.

11. Nikolelis D.P., Raftopoulou G., Nikoleli G.-P., Simantiraki M. Stabilized lipid membrane based biosensors with incorporated enzyme for repetitive uses. Electroanalysis. 2006, 18, 2467-2474.

12. Nikolelis D.P., Raftopoulou G., Chatzigeorgiou P., Nikoleli G.-P., Viras K. Optical portable biosensors based on stabilized lipid membrane for the rapid detection of doping materials in human urine. Sens. Actuators $B$ Chem. 2008, 130, 577-582. 
13. Nikoleli, G.-P.; Israr, M.Q.; Tzamtzis, N.; Nikolelis, D.P.; Willander, M.; Psaroudakis, N. Structural characterization of graphene nanosheets for miniaturization of potentiometric urea lipid film based biosensors. Electroanalysis 2012, 24, 1285-1295.

14. Bratakou, S.; Nikoleli, G.-P.; Nikolelis, D.P.; Psaroudakis, N. Development of a potentiometric chemical sensor for the rapid detection of carbofuran based on air stable lipid films with incorporated calix[4]arene phosphoryl receptor using graphene electrodes. Electroanalysis 2015, 27, 2608-2613.

15. Bratakou, S.; Nikoleli, G.-P.; Siontorou, C.G.; Nikolelis, D.P.; Tzamtzis, N. Electrochemical biosensor for naphthalene acetic acid in fruits and vegetables based on lipid films with incorporated auxin-binding protein receptor using graphene electrodes. Electroanalysis 2016, 28, 21712177.

16. Karapetis, S.; Nikoleli, G.-P.; Siontorou, C.G.; Nikolelis, D.P.; Tzamtzis, N.; Psaroudakis, N. Development of an electrochemical biosensor for the rapid detection of cholera toxin based on air stable lipid films with incorporated ganglioside GM1 using graphene electrodes. Electroanalysis 2016, 28, 1584-1590.

17. Bratakou, S.; Nikoleli, G.-P.; Siontorou, G.C.; Nikolelis, D.P.; Karapetis, S.; Tzamtzis, N. Development of an electrochemical biosensor for the rapid detection of saxitoxin based on air stable lipid films with incorporated Anti-STX using graphene electrodes. Electroanalysis 2017, 29, 990-997. 
18. Nikolelis, D. P., Simantiraki, M., Siontorou, G. C., Toth, K. Flow injection analysis of carbofuran in foods using air stable lipid film based acetylcholinesterase biosensor, Anal. Chim. Acta 2005, 537, 169-177

19. Nikolelis, D. P., Raftopoulou, G., Simantiraki, M., Psaroudakis, N., Nikoleli, G.-P., Hianik, T. Preparation of a selective receptor for carbofuran for the development of a simple optical spot test for its rapid detection using stabilized in air lipid films with incorporated receptor, Anal. Chim. Acta 2008, 620, 134-141

20. Nikolelis, D. P., Ntanos, N., Nikoleli, G.-P., Tampouris, K. Development of an electrochemical biosensor for the rapid detection of naphthalene acetic acid in fruits by using air stable lipid films with incorporated auxinbinding protein 1 receptor, Protein and Peptide Lett. 2008, 15, 789-794

21. Bratakou, S., Nikoleli, G-P. Siontorou, C.G., Karapetis, S., Nikolelis, D.P, Tzamtzis, N. Electrochemical biosensor for naphthalene acetic acid in fruits and vegetables based on lipid films with incorporated auxin-binding protein receptor using graphene electrodes, Electroanalysis 2016, 28, $2171-2177$

22. Nikoleli, G.-P.; Israr, M.Q.; Tzamtzis, N.; Nikolelis, D.P.; Willander, M.; Psaroudakis, N. Structural Characterization of Graphene Nanosheets for Miniaturization of Potentiometric Urea Lipid Film Based Biosensors. Electroanalysis 2012, 24, 1285-1295.

23. Nikoleli, G.-P., Siontorou, C.G., Nikolelis, D.P., Bratakou, S., Karapetis, S., Tzamtzis, N. Biosensors based on lipid modified graphene microelectrodes, Carbon 2017, 3(1), 9; doi:10.3390/c3010009 
24. Nikolelis, D. P., Raftopoulou, G., N. Psaroudakis, Nikoleli, G.-P. Development of an electrochemical chemosensor for the rapid detection of zinc based on air stable lipid films with incorporated calix4arene phosphoryl receptor. Int. J. Environ. Anal. Chem. 2009, 89, 211-222

25. Bratakou, S.; Nikoleli, G.-P.; Nikolelis, D.P.; Psaroudakis, N. Development of a potentiometric chemical sensor for the rapid detection of carbofuran based on air stable lipid films with incorporated calix[4]arene phosphoryl receptor using graphene electrodes. Electroanalysis 2015, 27, 2608-2613.

26. Nikolelis, D.P.; Andreou, V.G. Electrochemical transduction of interactions of atrazine with bilayer lipid membranes. Electroanalysis 2005, 8, 643-647.

27. Nikolelis, D.P.; Siontorou, C.G. Flow injection monitoring and analysis of mixtures of simazine, atrazine, and propazine using filter-supported bilayer lipid membranes (BLMs). Electroanalysis 1996, 8, 907-912.

28. Siontorou, C.G.; Nikolelis, D.P.; Tarus, B.; Dumbrava, J.; Krull, U.J. DNA biosensor based on self-assembled bilayer lipid membranes for the detection of hydrazines. Electroanalysis 1998, 10, 691-694.

29. Nikoleli, G.-P., Nikolelis, D.P., Tzamtzis, N. Development of an electrochemical biosensor for the rapid detection of cholera toxin using air stable lipid films with incorporated ganglioside GM1. Electroanalysis 2011, 23(9), 2182-2189.

30. Karapetis, S.; Nikoleli, G.-P.; Siontorou, C.G.; Nikolelis, D.P.; Tzamtzis, N.; Psaroudakis, N. Development of an electrochemical biosensor for the rapid detection of cholera toxin based on air stable 
lipid films with incorporated ganglioside GM1 using graphene electrodes. Electroanalysis 2016, 28, 1584-1590.

31. Michaloliakos, A.I.; Nikoleli, G.-P.; Siontorou, C.G.; Nikolelis, D.P. Rapid flow injection electrochemical detection of arochlor 1242 using stabilized lipid membranes with incorporated sheep anti-PCB antibody. Electroanalysis 2012, 24, 495-501.

32. Bratakou, S.; Nikoleli, G.-P.; Siontorou, G.C.; Nikolelis, D.P.; Karapetis, S.; Tzamtzis, N. Development of an electrochemical biosensor for the rapid detection of saxitoxin based on air stable lipid films with $\begin{array}{llll}\text { incorporated } & \text { Anti-STX } & \text { using }\end{array}$ electrodes. Electroanalysis 2017, 29, 990-997.

33. Nikolelis D.P., Pantoulias S. A minisensor for the rapid screening of sucralose based on surface-stabilized bilayer lipid membranes. Biosens Bioelectron. 2000, 5(9-10), 439-44.

34. Nikolelis D.P., Pantoulias S. Selective continuous monitoring and analysis of mixtures of acesulfame-K, cyclamate, and saccharin in artificial sweetener tablets, diet soft drinks, yogurts, and wines using filter-supported bilayer lipid membranes. Anal. Chem. 2001, 73(24), $5945-5952$. 
35. Chekashkina, K.V., Galimzyanov, T.R., Kuzmin, P.I. Akimov, S.A., Romanov, S.A., Pozmogova, G.E., Klinov, D.V., Bashkirov, P.V. Detection of DNA molecules in a lipid nanotube channel in the low ion strength conditions, Biochemistry (Moscow), Supplement Series A: Membrane and Cell Biology, 2017, 11(3), 217-224.

36. D'Souza, S.F., Kumar, J., Jha, S.K., Kubal, B.S., Immobilization of the urease on eggshell membrane and its application in biosensor, Mat. Sci. \& Engin. C 2013, 33, 850-854.

37. Trojanowicz, M., Miernik, A. Bilayer lipid membrane glucose biosensors with improved stability and sensitivity, Electr. Acta, 2001, 46(7), 10531061.

38. .Chen, H., Zheng, Y., Jian, C.-Y., Wu, H.-L., Shen, G.-L., Yu, R.-Q. An ultrasensitive chemiluminescence biosensor for cholera toxin based on ganglioside-functionalized supported lipid membrane and liposome, Biosens. \& Bioelectr., 2008, 24(4), 684-689.

39. Liu, N., Gao, Z., Zhou, H.Y., You, M. Detection of SEB gene by bilayer lipid membranes nucleic acid biosensor supported by modified patchclamp pipette electrode, Biosens. \& Bioelectr., 2007, 22(9-10), 23712376.

40. Xia, W., Li, Y., Wan, Y., Chen, T., Wei, J., Li, Y., Xu, S. ,Electrochemical biosensor for estrogenic substance using lipid bilayers modified by Au nanoparticles, Biosens. \& Bioelectr. 2010, 10(15), 2253-2258. 